\title{
Variantes Benignas do Eletrencefalograma
}

\author{
Délrio Façanha da Silva* Márcia Marques de Lima** \\ Renato Anghinah ${ }^{* *}$
}

\begin{abstract}
RESUMO
A identificaçăo de determinados achados eletrencefalográficos, que são considerados variantes benignas, í importante para que não sejam catalogados como anormais. Entre estes grafoelementos sem significado patológico no EEG, temos a descarga ritmica temporal média (conhecida como variante psicomotora), o complexo pontaonda $6 \mathrm{~Hz}$ (ponta-onda "fantasma"), as pontas positivas $6 / 14 \mathrm{~Hz}$, os paroxismos epiléticos benignos do sono, as "wicket spikes", a descarga paroxistica do "carrefour" e, mais recentemente, as pontas positivas $28 \mathrm{~Hz}$ o o potencial " $\mathrm{N}$ ". Todas essas variantes da normalidade precisam ser identificadas corretamente $\theta$ diferenciadas de padrōes anormais de significado diagnóstico em pacientes sintomáticos ou não.
\end{abstract}

\section{UNITERMOS}

Variantes Benignas, EEG
Pós-doutorado pela Faculdade de Medicina de Paris. Chefe do Setor de EEG da Disciplina de Neurologia da Escola Paulista de Medicina - SP.

** Médicos Estagiários do Setor de EEG da Escola Paulista de Medicina.
Durante a década de 1950 , o ritmo $m u$ era catalogado como um padrão eletrencefalográfico de significado duvidoso e muitas foram as associações deste ritmo com vários quadros clínicos, mas atualmente é aceito como um ritmo normal (Westmoreland, 1990). Ainda nesta época, outros grafoelementos no EEG foram surgindo e muitas divergências na literatura passaram a existir sobre o real significado de determinados achados, como as pontas positivas 6-14Hz (Gibbs e Gibbs, 1952). Nas décadas subseqüentes novos achados no EEG foram publicados, como a descarga paroxística do "carrefour" (Naquet et al., 1961, 1965), as "wicket spikes"(Reiher e Lebel, 1977)e, mais recentemente, as pontas positivas $28 \mathrm{~Hz}$ e o potencial " $N$ " (Reiher e Carmant, 1991). A identificação destes grafoelementos no EEG é importante para que não sejam catalogados como anormais, o que pode acarretar conseqüências imprevisíveis para os pacientes.

\section{DESCARGA RITMICA TEMPORAL MÉDIA}

Descrita inicialmente por Gibbs e Gibbs (1952) com o nome de variante psicomotora (VPSM), foi posteriormente denominada de theta temporal rítmico da sonolência (Chatrian et al., 1974), descarga rítmica temporal média (DRTM) (Lipman e Hughes, 1969; Arfel et al., 1978) e descarga padrão Gibbs (Tanarro e Lope, 1984). O termo proposto por Gibbs e Gibbs (1952), VPSM, foi escolhido pelo fato deste achado eletrencefalográfico ser semelhante ao observado na crise psicomotora, atualmente denominada de crise parcial complexa. Preferimos o termo DRTM, por descrever estritamente as características eletrencefalográficas, sem inferências clínicas como a VPSM, ou seja, destaca as suas três características marcantes no EEG, que são a forma de apresentação como uma descarga, a ritmicidade e a localização na região temporal média (Lipman e Hughes, 1969). Ao contrário da descarga epiléptica de localização temporal, a DRTM, assim como algumas das outras variantes benignas que serão citadas posteriormente (complexo ponta-onda $6 \mathrm{~Hz}$, espículas benignas do sono, "wicket spikes" e o potencial " $N$ "), não persistem nas fases profundas do sono não-REM. 
A DRTM caracteriza-se por um rítmo theta de início súbito com frequiência de $5-6 \mathrm{~Hz}$, de projeção na região temporal média, uni ou bilateral, podendo atingir as regiões vizinhas. A sua duração é variável, podendo ser de alguns segundos ou mais de $1 \mathrm{~h}$. A abertura e fechamento dos olhos e a foto-estimulação não inteferem nas suas características, pode surgir durante a prova da hiperpnéia, principalmente na sonolência e não nas fases III e IV do sono, o que a distingue do foco epiléptico, e raramente na vigília. A faixa etária mais comum de ser observada é no adulto jovem. Gibbs et al. (1963) encontraram a DRTM em 0,5\% de pacientes encaminhados para a realização de EEG por vários quadros clínicos e na série de Maulsby (1979) o achado foi de $2 \%$ entre adultos jovens normais. Atualmente, a DRTM não está associada às epilepsias, como era aceito logo nos primeiros anos de sua descrição, mas é considerada como um padrão benigno no EEG (Klass e Westmoreland, 1985).

\section{COMPLEXO PONTA-ONDA 6HZ}

Desde sua primeira publicação na década de 1950 (Walter, 1950, Gibbs e Gibbs, 1952), o complexo ponta-onda $6 \mathrm{~Hz}$ gerou muitas controvérsias sobre seu real significado. Atualmente é considerado como uma variante benigna da normalidade, não existindo evidências de associação com as epilepsias (Klass e Westmoreland, 1985). É também conhecido pela denominação de complexo ponta-onda "fantasma" devido à morfologia e à baixa amplitude da espícula, o que torna às vezes difícil sua visualização. Ao contrário da espícula, o componente lento do complexo é caracterizado por ondas theta de maior amplitude. Ocorre em surtos breves, geralmente com duração de 1 ou 2 segundos e raramente maior que 4 segundos. Comumente se apresenta de forma difusa, bilateral e sincrona, podendo ser assimétrico ou com maior amplitude nas regiões anteriores ou posteriores. É um achado incomum, com incidência em torno de $2,5 \%$, sendo visto principalmente em adolescentes e adultos (Westmoreland, 1990).

Hughes (1980) subdividiu o complexo ponta-onda $6 \mathrm{~Hz}$ em dois tipos. O primeiro foi chamado de padrão "VAAH" (presente na vigília, com alta amplitude, predominando nas regiões anteriores e em homens); o segundo, recebeu a denominação de padrão "MOBS" (em mulheres, predominando nas regiões ocipitais, com baixa amplitude e na sonolência). Neste estudo, Hughes (1980) encontrou uma maior incidência de crises epilépticas nos pacientes com o padrão "VAAH" e de sintomas neurovegetativos genéricos como cefaléia, tontura, vertigem, náuseas, vômitos, dor abdominal e lipotimia, naqueles com o padrão "MOBS". Entretanto, nem sempre estes dois clássicos tipos são encontrados, ocorrendo padrões intermediários entre eles.
O diferencial do complexo ponta-onda $6 \mathrm{~Hz}$, considerado uma variante benigna, deve ser feito com as descargas epilépticas caracterizadas pelos complexos ponta-onda. $\mathrm{O}$ complexo ponta-onda $6 \mathrm{~Hz}$ ou complexo ponta-onda "fantasma", apresenta a espícula de baixa amplitude, ocorre preferencialmente durante a sonolência e desaparece nas outras fases do sono, enquanto as descargas epilépticas em forma de complexos ponta-onda podem permanecer ou mesmo aumentar sua freqüência nas fases mais profundas do sono lento (Klass e Westmoreland, 1985).

\section{PONTAS POSITIVAS 6/14HZ E 28HZ}

As pontas positivas $6 / 14 \mathrm{~Hz}$ foram descritas há mais de 40 anos (Gibbs e Gibbs, 1951) e são, também, conhecidas como "ctenóides" ou surtos positivos $6 / 14 \mathrm{~Hz}$. Os surtos duram geralmente menos que um segundo, uni ou bilaterais, e são mais evidentes nas regiões temporais posteriores, sobretudo na sonolência. Podem ser visualizadas no EEG de indivíduos normais a partir dos 3 anos de idade, com uma maior incidência (10-58\%) ao redor dos 13 anos e tornandose mais raras com o avanço da faixa etária (Klass e Westmoreland, 1985).

No passado, foram associadas à diferentes quadros clínicos, como as epilepsias (Gibbs e Gibbs, 1951), os comas hepáticos (Poser e Ziegler, 1958; Silverman, 1964), a síndrome de Reye (Yamada et al., 1976, 1977) e comas em geral (Ford e Freeman, 1982; Falcon, 1986). Atualmente é considerada como uma variante benígna e sem nenhuma relação específica com qualquer quadro clínico (Drury, 1989).

Mais recentemente, foi publicado pela primeira vez na literatura, uma outra variante provavelmente benigna (Reiher e Carmant, 1991). São as minúsculas pontas positivas $28 \mathrm{~Hz}$. Elas ocorrem principalmente em crianças e raramente nos adolescentes. Surgem na sonolência, raramente na vigília, e podem ser uni ou bilaterais, síncronas ou assíncronas. Devido à sua baixíssima amplitude podem passar desapercebidas durante a análise do EEG. Apesar de uma publicação única, foi considerada por Reiher e Carmant (1991) como um achado eletrencefalográfico sem significado patológico.

\section{ESPICULAS BENIGNAS DO SONO}

As espículas benignas do sono (EBS) (Klass e Westmoreland, 1985) são também conhecidas como pequenas pontas (Gibbs e Gibbs, 1951) e paroxismos epileptiformes benignos do sono (White et al., 1977). Este último termo caiu em desuso pelo fato de ter sido verificado em vários estudos que não existe nenhuma associação específica com 
as epilepsias, como demonstraram White et al. (1977), que observaram as EBS em $24 \%$ de indivíduos normais e em $20 \%$ de pacientes com diferentes quadros clínicos. Estes dados demonstram que a identificação das EBS em diferentes diagnósticos clínicos é apenas uma associação casual.

As EBS ocorrem principalmente em adultos, sobretudo nas fases I e II do sono não-REM e são melhor visualizadas nas regiões temporais. Geralmente apresentam uma amplitude inferior a $50 \mathrm{uV}$, são de curta duração (menor que $50 \mathrm{mseg}$.) e podem ser uni ou bilaterais. Ao contrário da descarga epiléptica do foco temporal, as EBS não alteram a atividade elétrica cerebral de base circunvizinha, não apresentam a onda lenta da pós-descarga, apresentam menor amplitude, menor duração, diminuem em número ou desaparecem nas fases III e IV do sono não-REM.

\section{"WICKET SPIKES"}

O padrão "Wicket" foi melhor estudado por Reiher e Lebel (1977), através da análise de traçados eletrencefalográficos de 4.458 pacientes em sono e vigília, ao longo de 7 anos. Neste estudo, apenas 39 pacientes apresentaram as "Wicket Spikes" (WS) (cerca de 0,9\% do total de pacientes).

As WS caracterizam-se por predominar nas regiōes temporais anterior e média, sob a forma de ondas arciformes, de projeção uni ou bilateral, embora possa haver predomínio de um lado sobre o outro. Apresentam uma frequiência que varia entre $6 \mathrm{e} 11 \mathrm{~Hz}$, podendo ser encontradas agrupadas em surtos ou isoladas (Fig. 1). Apresentam entre 60 e $210 u V$ de amplitude, são monofásicas, mais facilmente reconhecidas durante a sonolência e raramente são observadas na vigília. Ocorrem em indivíduos adultos, geralmente acima dos 30 anos, e principalmente em idosos.

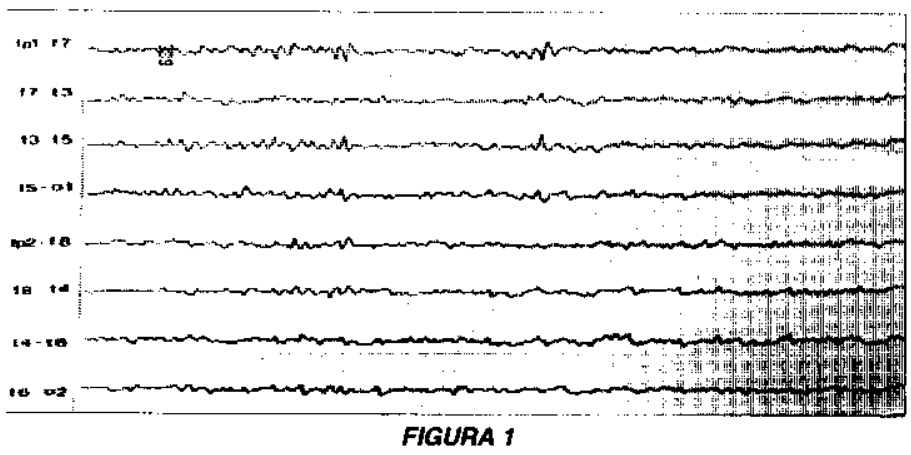

Embora possam ocorrer associadas às descargas epilépticas no traçado eletrencefalográfico, não são consideradas como fenômeno epiléptico, pois são freqüentemente vistas em associação casual com diferentes quadros clínicos, como vertigem, cefaléia, "tic douloreux", sincope e outros, com os quais não tem qualquer relação (Reiher e Lebel, 1977); Klass e Westmoreland, 1985).
Asokan et al. (1987), relacionaram o padrão WS com a existência de doença cérebro-vascular, o que explicaria sua maior incidência em indivíduos adultos, principalmente acima dos 50 anos. Por outro lado, Hughes e Olson (1981) observaram uma alta incidência $(70 \%)$ de sintomas neurovegetativos nos pacientes com as WS no eletrencefalograma, principalmente no sexo feminino (62\%). Nossa impressão é que as WS são apenas uma representação do ritmo "en arceau", ou seja, são benignas e não apresentam nenhum significado patológico.

Pode ser difícil diferenciar as WS de descargas epilépticas tipo pontas, especialmente quando isoladas. Entretanto, Reiher e Lebel (1977) apontam como parâmetro valioso e cardial, saber perceber que surtos ou "clusters" de WS invariavelmente precedem as WS isoladas e que, ao contrário do foco epiléptico de localização temporal, desaparecem nas fases mais profundas do sono lento. Além disso, as WS não alteram a atividade elétrica cerebral de base e não apresentam a onda lenta da pós-descarga do foco epiléptico.

\section{DESCARGA PAROXISTICA DO "CARREFOUR"}

Desde a primeira publicação deste padrão EEG por Naquet et al. (1961, 1965) são raras as publicações na literatura à respeito deste grafoelemento (Westmoreland $\mathrm{e}$ Klass, 1981; Miller et al., 1985; Thomas et al., 1992), com uma incidência de 0,02-0,045\% (Tanarro e Lope, 1984). É também conhecido como descarga paroxística padrão Naquet (Tanarro e Lope, 1984) e descarga eletrográfica rítmica subclínica do adulto (DERSA) (Westmoreland e Klass, 1981).

Naquet et al. (1961), e Gastaut e Naquet (1966), descreveram este padrão EEG como uma descarga paroxística por ondas agudas de início súbito, que progressivamente aumenta de frequiência, evoluindo para uma descarga eletrográfica rítmica subclínica de $6-8 \mathrm{~Hz}$, associadas com ondas agudas (Fig. 2), podendo apresentar uma duração superior a 1 minuto, com final abrupto e imediata normalização do traçado. Esta descarga pode ser focal ou generalizada, mas

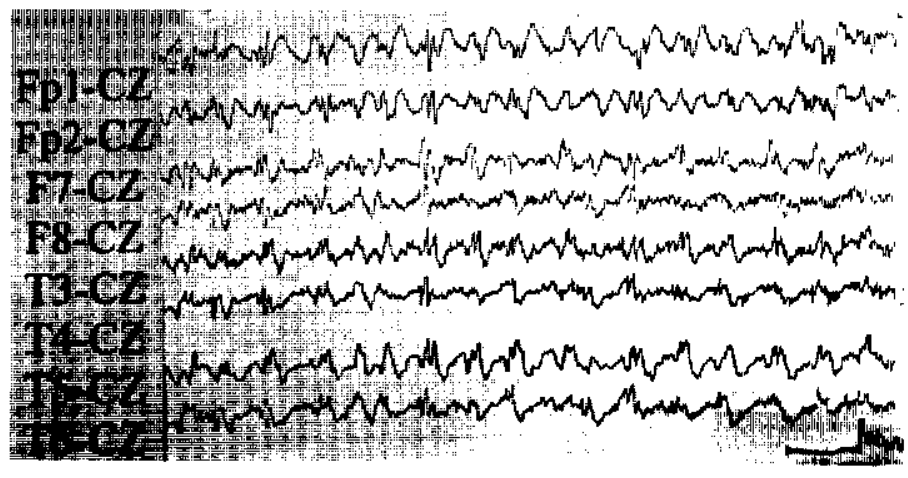

FIGURA 2 
predominando na região do "carrefour" (região temporoparieto-occipital) e ocorre tanto na vigília como no sono.

A DERSA geralmente persiste nos EEG evolutivos (Naquet et al., 1961, 1965; Westmoreland e Klass, 1981; Tanarro e Lope, 1984; Thomas et al., 1992), e raramente isto não é observado, o que justificou, recentemente, a apresentação de um caso clínico por nosso Setor durante um evento científico (XIII Congresso Brasileiro de Neurofisiologia Clínica, Belo Horizonte - MG, 12-15 de outubro de 1993).

A gênese da DERSA ainda não está bem definida. A teoria proposta por Naquet et al. (1961) relaciona a DERSA a mecanismos hipóxico-isquêmicos na crítica região do "carrefour" em pacientes idosos. Tannarro e Lope (1984) publicaram um estudo de seis pacientes com idade entre 31 e 50 anos, que apresentavam nos seus EEG, tanto a DERSA, como a variante psicomotora, sugerindo que estes dois tipos de descargas são análogas e podem representar um fenômeno idêntico, o que contraria a teoria de Naquet et al. (1961), assim como, o trabalho de Thomas et al. (1992), que em um estudo com tomografia computadorizada por emissão de photon ("Spect") concluíram que a DERSA não pode ser considerada como um padrão epiléptico e deve estar relacionada a mecanismos vasculares.

Em suma, a DERSA é considerada um padrão EEG benigno e de significado incerto (Westmoreland, 1990) e é importante não interpretar este padrão EEG como um fenômeno epiléptico.

\section{POTENCLAL " $N "$}

O potencial " $N$ ", juntamente com as pontas positivas $28 \mathrm{~Hz}$, é o mais recente padrão eletrencefalográfico descrito por Reiher e Carmant (1991). Caracteriza-se por uma única espícula de polaridade negativa e de baixa amplitude, que se acompanha por ondas theta de aproximadamente $200 \mathrm{uV}$ de amplitude e com a morfologia de um " $N$ ". Frequientemente surge no EEG em conjunto com as pontas positivas $6 / 14 \mathrm{~Hz}$. Pode se apresentar de forma uni ou bilateral, síncrono ou assíncrono, mais freqüentemente na sonolência que na vigília e raramente nas fases profundas do sono (Reiher e Carmant, 1991). Este novo achado eletrencefalográfico foi observado somente em crianças e adolescentes com diferentes quadros clínicos, como cefaléia, distúrbio de conduta, síncope, trauma crânio-encefálico e convulsão (Reiher e Carmant, 1991).

Na literatura por nós pesquisada, não encontramos novos relatos à respeito do potencial " $N$ ". Recentemente tivemos a oportunidade de apresentar em um congresso (XIII Congresso Brasileiro de Neurofisiologia Clínica, Belo Horizonte - MG, 12-15 de outubro de 1993), os casos de duas crianças com cefaléia tensional, que apresentavam este recém descrito achado eletrencefalográfico. Durante este evento científico, propusemos a denominação de Complexo
" $N$ " no lugar de potencial " $N$ ", por acharmos este termo mais abrangente em relação à sua própria morfologia. Além disso, não consideramos haver qualquer relação com os quadros clínicos apresentados pelos pacientes.

Apesar de raros estudos à respeito do complexo " $N$ ", o real significado deste novo achado eletrencefalográfico parece ser de um padrão eletrencefalográfico benigno. No trabalho de Reiher e Carmant (1991), estes autores sugerem ser uma variante da normalidade devido aos quadros clínicos encontrados e a frequiente associação com as pontas positivas, que já apresentam uma associação bem definida na literatura como uma variante benigna do EEG (Lombroso et al., 1966; Drury, 1989).

O reconhecimento deste novo achado no EEG é importante para evitarmos que seja confundido com os complexos ponta-onda atípicos e, mesmo, com os complexos $\mathrm{K}$ do sono.

\section{Comentários finais}

Além das variantes benignas aqui relatadas, outras variantes da normalidade são descritas, como as ondas lentas posteriores da juventude (onda "PI" ou OLIP), a hipersincronia hipnagógica, ritmos de falha óssea, a variante alfa rápida e lenta, os ritmos lentos temporais e os surtos de theta pontiagudos da linha média. Todas essas outras variantes já fazem parte dos capítulos de livros sob o título "EEG normal", daí não termos tecido comentários à respeito. Achamos que em um futuro bem breve o tema "Variantes Benígnas do EEG" não mais constará como um capítulo à parte nos tratados de eletrencefalografia e serão todas englobadas sob um único título, o EEG normal.

\section{SUMMARY \\ Benign Variants of the EEG}

The recongnition of some EEG patterns known as benign variants is important to avoid misconsctruction with abnormal pattems. These graphoelements without pathologic meaning in the EEG are the rhythmic midtemporal discharge (psychomotor variant). the $6 \mathrm{~Hz}$ spike-and-slow waves (phantom spike-wave), the $14 / 6 \mathrm{~Hz}$ positive bursts, the benign sporadic sleep spikes, the wicket spikes, the "décharges paroxystiques du carrefour" and more recently the $28 \mathrm{~Hz}$ positive bursts and the $\mathrm{N}$-shape potential. All these normal variants need to be identified correctly and differentiated from abnormal pattems of diagnostic significance in symptomatic or asymptomatic patients.

\section{Key Words}

Benign Variants, EEG 


\section{Bibliografia}

1 ARFEL, G.; LEONARDON, N.; BUREAU, M; ISMAN, M.L.; NAQUET, R. - Décharges életriques temporales inexpliquées. Rev EEG Neurophysiol., 3:335-340, 1978.

2 ASOKAN, G.; PAREJA, J.; NIEDERMEYER, E. - Temporal slow and sharp EEG activity and cerebrovascular disorder. Clin Electroenceph, 18:201-210, 1987.

3 CHATRIAN, G.E.; BERGAMINI, L.; DONDEY, M.; KLASS, D.W.; LENNOX-BUCHTAL, M.; PETERSEN, I. - A glossary of terms most commonly used by clinical electroenchephalographers. Electroenceph $\mathrm{Cl}$ Neurophysiol, 37:348-358, 1974.

4 DRURY, I. - 14-and-6Hz positive bursts in childhood encephalopathies. Electroenceph CI Neurophysiol, 72:479-485, 1989.

5 FALCON, J. - 14 and/or $6 \mathrm{~Hz}$ positive bursts recorded in comatose patients. Electroenceph CI Neurophysiol, 64:24p, 1986.

6 FORD, R.G.; FREEMAN, A.M. - Positive spike bursts in a comatose adult. Electroenceph Cl Neurophysiol, 53:29p, 1982.

7 GASTAUT, H; NAQUET, R. - Étude électroencéphalographique de l'insuffisance circulatoire cérébral (ICC). Dans: Symposium International sur la Circulation Cérébrale. Sandoz, Paris, 1966, p163-191.

8 GIBBS, E.L.; GIBBS, F.A. - Electroencephalography evidence of thalamic and hypothalamic epilepsy. Neurology, 1:136-144, 1951.

9 GIBBS, E.L.; GIBBS, F.A. - Atias of Electroencephalography, Vol. 2, Cambridge, $2^{\mathrm{a}}$ ed., Addison-Wesley, Reading, MA, 1952, p356-374.

10 GIBBS, F.A.; RICH, C.L.; GIBBS, E.L. - Psychomotor variant type of seizure discharge. Neurology (Minneap.), 13:991-998, 1963.

11 HUGHES, J.R. - Two forms of the $6 / \mathrm{sec}$ spike and wave complex. Electroenceph Cl Neurophysiol, 48:535-550, 1980.

12 HUGHES, J.R.; OLSON, S.F. - An investigation of eight different types of temporal discharges. Epilepsia, 22:421-435, 1981.

13 KLASS, D.W.; WESTMORELAND, B.F. - Nonepileptogenic epileptiform electroencephalographic activity. Ann Neurol, 18:627-635, 1985.

14 LIPMAN, I.J.; HUGHES, J.R. - Rhythmic mid-temporal discharges. An electroclinical study. Electroenceph Cl Neurophysiol, 27:43-47, 1969.

15 LOMBROSO, C.T.; SCHWARTZ, I.H.; CLARK, E.M.; MUENCH, H.; BARRY, J. - Ctenoids in healthy youths: controled study of 14-and-6per-second positive spiking. Neurology, 16:1152-1158, 1966.

16 MAULSBY, R.L. - EEG patterns of uncertain diagnostic significance. In: Current Practice of Clinical Electroencephalography. Klass, D.W. \& Pedley, D.D. (Eds), Raven Press, New York, 1979, p411-419.

17 MILLER, C.R.; WESTMORELAND, B.F.; KLASS, D.W. - Subclinical rhythmical EEG discharges of adults (SREDA): further observations. Am J EEG Technol, 25:217-224, 1985.
18 NAQUET, R.; LOUARD, C.; RHODES, J.; VIGOUROUX, M. - A propos de certaines décharges paroxystiques du carrefour pariéto-temporooccipital. Leur activation par l'hypoxie. Rev Neurol, 105:203-207, 1961.

19 NAQUET, R.; FRANCK, G.; VIGOUROUX, M. - Données nouvelles sur certaines décharges paroxystiques du carrefour pariéto-temporo-occipital, rencontrées chez l'homme. ZbI Neurochir, 25:153-180, 1965.

20 POSER, C.M.; ZIEGLER, D.K. - Clinical significance of 14 and 6 per second positive spike complexes. Neurology, 6:903-912, 1958.

21 REIHER, J.; CARMANT, L. - Clinical correlates and electroencephalographic characteristics of two additional patterns related to 14 and 6 per second positive spikes. Can J Neurol Sci, 16:488-491, 1991

22 REIHER, J.; LEBEL, M. - Wicket spikes: clinical correlates of a previously undescribed EEG pattern. J Can Scienc Neurol, 46:40-47. 1977.

23 SILVERMAN, D. - 14 and 6 per second positive spike pattern in a patient with hepatic coma. Electroenceph CI Neurophysiol, 16:395-398, 1964.

24 TANARRO, F.J.H.; LOPE, E.S. - EEG subclinical paroxysmal thythmic activity. Rev EEG Neurophysiol, 14:9-15, 1984.

25 TANARRO, F.J.H.; LOPE, E.S. - Subclinical paroxysmal rhythmic activity. Electroenceph $\mathrm{Cl}$ Neurophysiol, 58:5P, 1984.

26 THOMAS, P.; MIGNECO, O.; DARCOURT, J.; CHATEL, M. - Single photon emission computed tomography study of subclinical rhythmic electrographic discharge in adults. Electroenceph $\mathrm{Cl}$ Neurophysiol, 83:223-227, 1992.

27 WALTER, W.G. - Epilepsy. In: Electroencephalography. Hill, D. \& Parr, G. (Eds). MacDonald \& Company, London, 1950, p228-272.

28 WESTMORELAND, B.F.; KLASS, D.W. - A distinctive rhythmic EEG discharge of adults. Electroenceph $\mathrm{Cl}$ Neurophysiol, 51:186-191, 1981.

29 WESTMORELAND, B.F. - Benign EEG variants and patterns of uncertain significance. In: Current Practice of Clinical EEG. Daly, D.D. and Pedley, T.A. (Eds), 2nd Edition, Raven Press, New York, 1990, p243252.

30 WHITE, J.C.; LANGSTON, J.W.; PEDLEY, T.A. - Benign epileptiform transients of sleep. Clarification of the small sharp spike controversy. Neurology (Minneap.), 27:1061-1068, 1977

31 YAMADA, T.; TUCKER, R.P.; KOOI, K.A. - 14 and 6 c/sec. positive bursts in comatose patients. Electroenceph CI Neurophysiol, 40:645653, 1976.

32 YAMADA, T.; YOUNG, S.; KIMURA, J.; - Significance of positive spike bursts in Reye's syndrome. Arch Neurol, 34:376-380, 1977. 